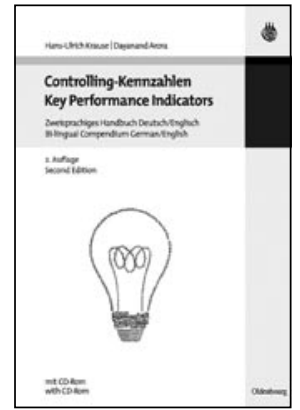

Hans-Ulrich

Krause,

Dayanand Arora

\section{Controlling-Kennzahlen Key Performance Indicators}

\author{
2. Aufl., Oldenbourg \\ München 2010 \\ 710 Seiten, $54,80 €$ \\ ISBN: 978-3-486-59690-8
}

Kennzahlen - sie bestimmen einen großen Teil des Wirtschaftsgeschehens. Ohne sie gäbe es wohl auf Bilanzpressekonferenzen und Aktionärshauptversammlungen weitaus weniger zu erzählen - was der eine oder andere sicherlich begrüßte. Dennoch, was seit jeher ein wesentlicher Bestandteil der externen Berichterstattung ist, findet vermehrt auch bei der internen Steuerung von Organisationen seine Anwendung. Klassischerweise besitzen sie hier zwei wesentliche Funktionen: zum einen soll ihre Bereitstellung die Entscheidungsfindung erleichtern und verbessern, zum anderen werden sie häufig zur Leistungsmessung und -beurteilung herangezogen. Nicht selten hängen von ihnen Bonuszahlungen oder auch Beförderungen ab. Kennzahlen, zumeist durch das Controlling bereitgestellt und in aggregierter Form als Key Performance Indicators kurz KPIs - benannt, trifft man in zunehmender Fülle in der unternehmerischen Praxis. Genau dieser Vielfalt möchten sich Hans-Ulrich Krause, Professor für Betriebswirtschaftslehre mit dem Schwerpunkt Controlling/Rechnungswesen, und Dayanand Arora, Professor für Betriebswirtschaftslehre mit dem Schwerpunkt Finanz- und Rechungswesen, mit ihrer Kennzahlen-Übersicht annehmen. Beide Professoren sind an der Hochschule für Technik und Wirtschaft in Berlin tätig und begegnen der Fülle an möglichen Kennzahlen mit einer gut nachvollziehbaren Struktur, die sich an den vier BasisPerspektiven der Balanced Scorecard orientiert. Dabei werden die insgesamt 158
Kennzahlen der Finanz-, Kunden-, Prozess- und Personal-/Innovations-Perspektive zugeordnet und ausführlich besprochen. Eine nützliche Besonderheit dieses Handbuchs ist seine Zweisprachigkeit. Jede der 355 Seiten steht einmal in deutscher, einmal in englischer Sprache zur Verfügung. Dies ist insbesondere von Vorteil, ist doch ein großer Teil der in Unternehmen verwendeten Kennzahlen mit englischen Namen versehen.

Jede der Beschreibungen erfolgt nach einem einheitlichen Schema, was dem Verständnis und der Übersichtlichkeit deutlich zu Gute kommt. Die Erklärungen umfassen zunächst eine kurze Illustration der generellen Fragestellung, der Analytical Question, die der Kennzahl zu Grunde liegt und welche diese nach Möglichkeit beantworten soll. Darauf folgt eine meist an eindeutigen Formeln angelehnte Definition der Kennzahl, welche gegebenenfalls noch durch ergänzende Kommentare näher erläutert wird. So wird die jeweilige Dimension genannt, in der die Kennzahl anzugeben ist. Weiter sind mögliche Bezugszeiträume oder kurze Beispiele mit aufgenommen. Anschließend wird in einem Absatz auf die Ermittlung/ Herleitung, respektive die Calculation/ Derivation, eingegangen. Konkret wird hier beschrieben, aus welchen Quellen, etwa der Bilanz, der GuV oder der internen Informationssystemen, die notwendigen Informationen für die jeweilige Kennzahl gewonnen werden können. Der betriebswirtschaftliche Aussagewert und die Größenordnung, die Interpretation and Typical Range, stehen nachfolgend im Mittelpunkt. Dabei wird versucht, dem Leser ein Gespür für die Aussagefähigkeit einer Kennzahl zu vermitteln. Der pädagogische Wert ist dabei nicht zu unterschätzen. Kennzahlen können oftmals eine gute Hilfestellung bieten und Transparenz fördern. Gleichzeitig dürfen sie jedoch nicht als absolute, in unter allen Umständen immer gültige Wahrheit verstanden werden. Immer gilt es, den jeweiligen Kontext und die getroffenen Annahmen zu berücksichtigen. Der darauf folgende Abschnitt behandelt die Handlungsanregungen, die englisch etwas treffender mit Useful Suggestions beschrieben werden. Darin werden mögliche Wege dargestellt, wie die jeweilige Kennzahl von Interesse beeinflusst und gesteuert werden kann. Häufig enthalten sind dabei Hinweise auf Methoden und Verfahren, die dies erleichtern können. Den Abschluss einer jeden Kennzahlenbesprechung bildet die Rubrik Verwandte Kennzahlen/ Zusätzliche Anmerkungen, Related Ratios/Addtional Notes. Besprochen werden hier häufige Verwechslungen oder Indikatoren die derselben „Familie“ zugehörig sind und sich mit einer ähnlichen Fragestellung beschäftigen.

Besonders gewonnen hat die zweite Ausgabe durch die Dreingabe einer CD, auf der sich für alle Kennzahlen ein Excelbasiertes Kalkulationsprogramm befindet. Zudem wurden - um die Nutzung zu vereinfachen - 20 SMARTE (Specific, Measureable, Achievable, Relevant und Time-bound) Kennzahlen zum Einstieg ausgewählt. Diese setzen sich aus je 5 Kennzahlen der verschiedenen Perspektiven der Balanced Scorecard zusammen.

Zusammenfassend weist dieses Buch den Charakter eines umfassenden Nachschlagewerks auf. Durch die Erläuterung der Kennzahlen und der dahinterliegenden Ursache-Wirkungs-Beziehungen liefert dieses Werk umfassende Informationen $\mathrm{zu}$ modernen Management-Methoden. Hiervon profitieren insbesondere Praktiker. Für diese dürfte auch die zweisprachige Ausführung den größten Nutzen stiften, welche den internationalen Austausch erleichtert und eine bessere Kommunikation ermöglicht. Gleichzeitig wird der Aspekt der Zweisprachigkeit auch den Studierenden und Lehrende helfen, im Dschungel der Controlling-Kennzahlen den Überblick zu behalten.

Fabian Kleinschmit, Vallendar 